\title{
Adrenal function in patients with community-acquired pneumonia
}

\author{
S. Gotoh*, N. Nishimura*, O. Takahashi ${ }^{*}$, H. Shiratsuka ${ }^{\star}$, H. Horinouchi*, H. Ono*, \\ N. Uchiyama* and N. Chohnabayashi*
}

ABSTRACT: Adrenal insufficiency is believed to occur frequently in severe sepsis and septic shock. The aim of the present study was to determine whether adrenal function is also related to the severity of community-acquired pneumonia (CAP).

In total, 64 Japanese patients with CAP were consecutively enrolled in the present study, which was carried out during 2005-2006. Serum adrenocorticotropic hormone (ACTH) and cortisol were measured in each subject, as was the response of cortisol secretion when $250 \mu \mathrm{g}$ of cosyntropin was administered. Analyses were performed comparing these values with the score calculated according to the Pneumonia Patient Outcomes Research Team (PORT) cohort study, the number of in-hospital deaths and the length of hospital stay.

As the PORT score increased, serum ACTH and cortisol also increased, while the response of cortisol secretion to the administration of cosyntropin decreased. In the analysis by receiver operating characteristic curves, adrenal dysfunction was related significantly to both the number of in-hospital deaths and the length of hospital stay.

Adrenal dysfunction was shown to correlate with the Pneumonia Patient Outcomes Research Team score and the clinical outcomes, while adrenal insufficiency defined by the cosyntropin stimulation test was rare in the present study.

KEYWORDS: Adrenal insufficiency, adrenocorticotropic hormone, community-acquired pneumonia, cortisol

everal studies have revealed that a physiological dose of steroids is an effective therapy for patients with septic shock [13] and that patients with septic shock are lacking cortisol secretion to maintain the normal adrenal function [4-7]. This status has been called "relative adrenal insufficiency" [4, 8], a concept that describes the necessity of steroids for critically ill patients. However, the recently published results of the Costicosteroid Therapy of Septic Shock (CORTICUS) study [9] have shown that hydrocortisone therapy did not improve the survival of patients with septic shock in groups of patients who did or did not have a response to a corticotropin stimulation test. In addition, hydrocortisone therapy was reported to increase the relative risk ratio of complications, such as new sepsis and septic shock [9].

Although researchers have been interested in the adrenal function in critically ill patients with severe sepsis and septic shock, only a few small studies have investigated the relationship between adrenal function and the severity of

For editorial comments see page 1150. community-acquired pneumonia (CAP), even though the disease has a good scoring system for determining severity. In some papers, relative adrenal insufficiency was reported to occur in a high proportion of patients with severe CAP [10, 11]. CONFALONIERI et al. [12] demonstrated that a physiological dose of steroids may be beneficial for severe CAP in a randomised control study of steroid administration for patients with severe CAP, although the adrenal function of the patients was not shown. In addition, OPPERT et al. [13] reported two separate effects of the treatment with low-dose hydrocortisone in patients with early hyperdynamic septic shock: a hemodynamic effect, which is related to endogenous cortisol levels; and an immunomodulatory effect, which is independent of adrenal function. The present authors hypothesised that there might be some relationship between adrenal function and the severity of CAP. Therefore, the standard measures of adrenal function, i.e. serum cortisol, serum adrenocorticotropic hormone (ACTH) and the response of cortisol secretion to the administration of $250 \mu \mathrm{g}$ of cosyntropin, were examined and compared with the severity of CAP. The relationship of these measures with the

\section{AFFILIATIONS}

Depts of *Respiratory Medicine

${ }^{\#}$ General Internal Medicine, St

Luke's International Hospital, Tokyo, and

"Dept of Anaesthesiology, Fukui Red

Cross Hospital, Fukui, Japan.

\section{CORRESPONDENCE}

S. Gotoh

Akashi-cho 9-1

Chuo-ku

Tokyo

104-8560

Japan

Fax: 81335440649

E-mail: gotoshin@luke.or.jp

Received:

October 142007

Accepted after revision

January 292008

SUPPORT STATEMENT

Financial support for this study was received from St Luke's Life Science Institute (Tokyo, Japan).

STATEMENT OF INTEREST

None declared.
European Respiratory Journal

Print ISSN 0903-1936

Online ISSN 1399-3003 
outcome of the number of in-hospital deaths and the length of hospital stay were also investigated. Recently, CHRIST-CRAIN et al. [14] have reported that cortisol levels could be used as predictors of severity and death. In the present study, the authors added the analysis of the relationship between serum ACTH level and the severity of pneumonia and evaluated the results of cosyntropin stimulation test. Additionally, the issue of the dysfunction of the hypothalamic-pituitary-adrenal (HPA) axis in patients with CAP will be discussed in the present study.

\section{MATERIAL AND METHODS Study population}

In total, 69 hospitalised patients (St Luke's International Hospital, Tokyo, Japan) with clinical and radiographical evidence of CAP were consecutively enrolled during the period October 2005-October 2006. Of these, seven patients died in the hospital and seven patients were transferred to other hospitals. The clinical course of the transferred patients could not be followed; therefore, patients who died or were transferred to other hospitals were excluded when the average length of hospital stay was calculated. Exclusion criteria included any of the following conditions: 1) nosocomial pneumonia; 2) severe immunosuppression; 3) acute burn injury; 4) pregnancy; 5) a condition for which steroids had been administered; 6) aged $<18$ yrs; 7) a major gastrointestinal bleed within 3 months of the current hospitalisation; or 8) recovery from Candida species at multiple sites. Patients or their legally authorised representatives signed the consent form approved by the Research Ethics Committee at St Luke's International Hospital. In total, five patients were excluded from the study: two had tuberculosis; one had idiopathic interstitial pneumonia; and two were taking corticosteroids regularly. No patients were prescribed etomidate or other medications that affect the HPA axis.

\section{Data collection}

All registered patients were assigned to five risk classes based on 20 clinical variables as indicated in the Pneumonia Patient Outcomes Research Team (PORT) cohort study [15]. A cosyntropin stimulation test was performed with $0.25 \mathrm{mg}$ of tetracosactrin on the morning after admission from 07:00 10:00 h. Blood samples were obtained immediately before the test (T0), and 30 (T30) and 60 (T60) min after injection, and were stored at $4{ }^{\circ} \mathrm{C}$. After centrifugation, the concentrations of ACTH (normal range $7-56 \mu \mathrm{g} \cdot \mathrm{dL}^{-1}$ ) and cortisol (normal range 6.2 $19.4 \mu \mathrm{g} \cdot \mathrm{dL}^{-1}$ ) were measured by immunoradiometric assay (ACTH immunoradiometric assay kit; Mitsubishi Chemical, Tokyo, Japan) or an automated ELISA (Roche Diagnostics Corporation, Tokyo, Japan). The cortisol response was defined as the difference between T0 and the higher value of the T30 and T60 concentrations. Antibiotics were administered intravenously for empirical therapy as recommended by the guidelines of the Japanese Respiratory Society [16]. The number of inhospital deaths and the length of hospital stay were recorded.

\section{Statistical analysis}

The present authors investigated the relationship between the score calculated from 20 clinical variables based on the PORT cohort study and the values of serum cortisol, ACTH, and the increase of serum cortisol after cosyntropin-stimulation with Spearman's rank correlation coefficient. The relationship between patients with positive blood culture and adrenal function was evaluated using the Mann-Whitney U-test, and the relationship between the types of pathogen and adrenal function was evaluated using ANOVA. Their correlation with the number of in-hospital deaths and the length of hospital stay was then investigated using receiver operating characteristic (ROC) curves.
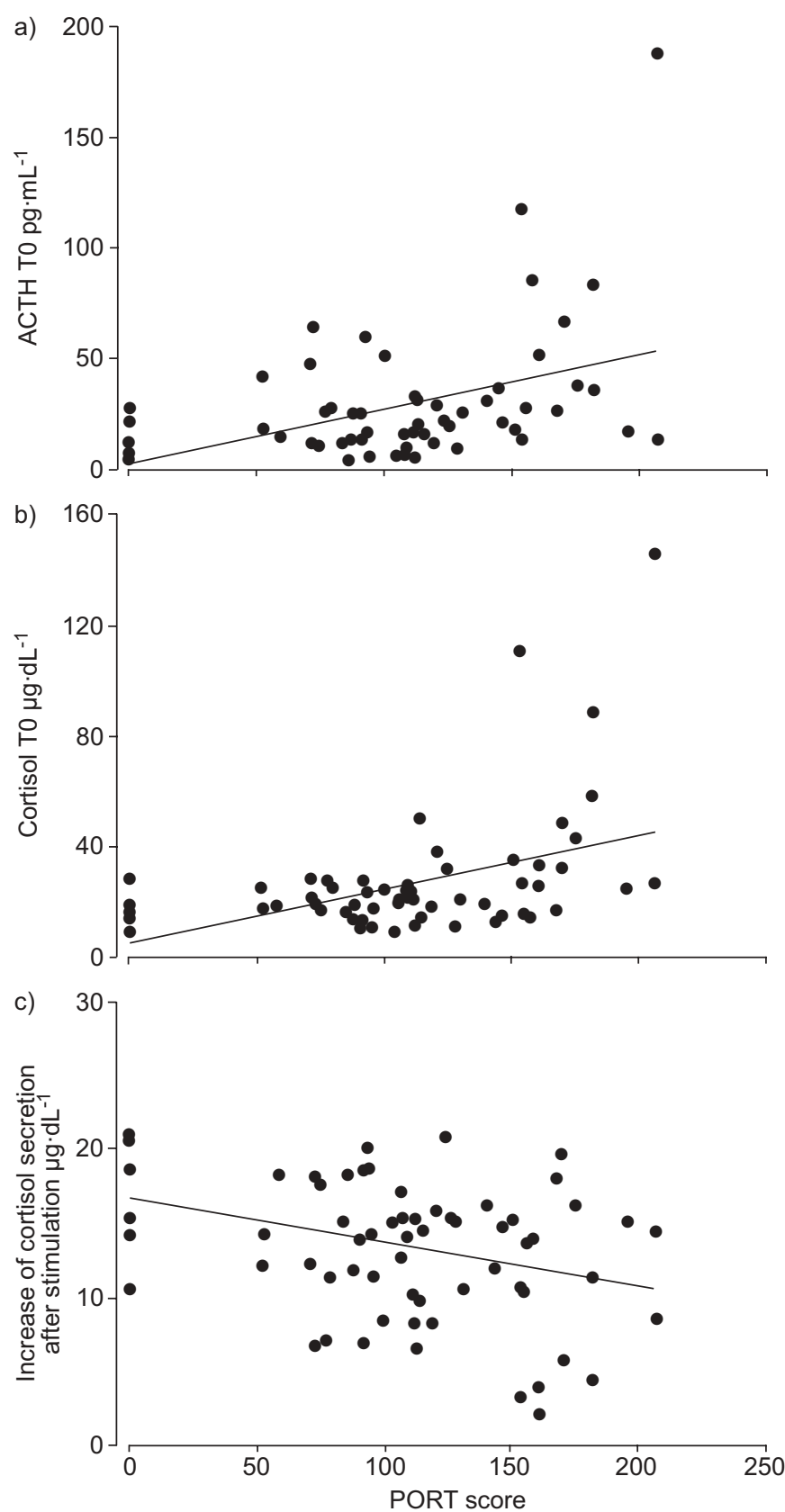

FIGURE 1. Correlation between adrenal function and severity of communityacquired pneumonia (CAP). The values of baseline concentration of a) serum adrenocorticotropic hormone (ACTH) immediately before cosyntropin administration (TO), b) serum cortisol TO, and c) increase in serum cortisol after cosyntropin stimulation were correlated with the Pneumonia Patient Outcomes Research Team (PORT) scores in 64 hospitalised patients with CAP. Spearman's rank correlation coefficients: a) $\rho=0.371, p=0.003$; b) $\rho=0.396, p=0.001$; c) $\rho=-0.273, p=0.029$ 


\section{RESULTS}

\section{Patients' characteristics}

A total of 64 patients with CAP were consecutively enrolled in the present study from October 2005-October 2006. Of these, 40 patients were male. The median age of the cohort was $77 \mathrm{yrs}$ and the median PORT score was 110. In order to treat CAP (table 1), 14 patients were receiving steroids, but the decision for steroid administration was independent of the result of the adrenal function test, except for the five patients with septic shock who were administered steroids after their clinical condition worsened. The corticosteroids dose was referred to previous studies $[12,17,18]$. The ACTH value before the administration of cosyntropin was missing for three patients due to failure to measure it. Almost all patients were initially treated empirically with intravenous $\beta$-lactum antibiotic agents in the present authors' hospital, according to the guidelines of the Japanese Respiratory Society [16]. When atypical pneumonia was suspected, minocycline, macrolide or fluoroquinolone was added. The causative pathogen was identified in 29 patients (e.g. Streptococcus pneumoniae was identified in sputum, blood culture or urinary antigen assay from nine patients), but the causative pathogen could not be identified in 35 patients because in some cases the culture specimen was taken after antibiotics were administered and in other cases sufficient sputum was not submitted (table 2). In the present study, seven patients died in hospital. The average length of the hospital stay excluding patients who died or were transferred to other hospitals was 17.7 days.

\section{Relationship between adrenal function and PORT score, positive blood culture and types of pathogens}

The secretion of serum cortisol, serum ACTH and the response of cortisol secretion to the administration of $250 \mu \mathrm{g}$ of ACTH was examined on admission. The median values of serum cortisol, serum ACTH and the increase of serum cortisol after stimulation were $21.3 \mu \mathrm{g} \cdot \mathrm{dL}^{-1}, 20 \mathrm{pg} \cdot \mathrm{mL}^{-1}$ and $14.3 \mu \mathrm{g} \cdot \mathrm{dL}^{-1}$, respectively (table 3 ). The correlation of these values with the PORT scores was evaluated. Serum cortisol and ACTH were significantly correlated with the PORT score (Spearman's correlation coefficients: $r=0.396, p=0.001$ and $r=371, p=0.003$, respectively), and the increase of serum cortisol after stimulation was negatively correlated with the PORT score $(r=-0.273$, $\mathrm{p}=0.029$; fig. 1). In addition, it was confirmed that serum cortisol and ACTH were significantly correlated with the PORT score in the subgroups of subjects that did not include the patients who were administered corticosteroids. There were four patients with positive blood culture; both serum cortisol and ACTH in these patients were significantly greater than in patients with negative blood culture (Mann-Whitney U-test, $p=0.004$ and 0.039 , respectively). There was no significant relationship between the types of pathogens and the PORT score (data not shown).

\section{Relationship between adrenal function and outcomes}

The present authors studied the correlations of serum ACTH, serum cortisol and the increase of serum cortisol after cosyntropin stimulation with the outcomes of the treatment for CAP. The number of in-hospital deaths and the length of hospital stay were regarded as the most important outcomes in the present study. These outcomes were analysed using ROC curves for serum ACTH, serum cortisol, the increase of serum cortisol after stimulation and the PORT score. Serum ACTH and the PORT score are good indicators of in-hospital deaths $(\mathrm{p}=0.007$ and 0.016 , respectively; fig. $2 \mathrm{a})$. The area under the ROC curve was 0.818 (95\% confidence interval (CI) 0.694 0.942) for serum ACTH, 0.650 (95\% CI 0.398-0.902) for serum cortisol and 0.782 (95\% CI 0.603-0.960) for the PORT score. Specificity $>90 \%$ was achieved for $>56 \mu \mathrm{g} \cdot \mathrm{dL}^{-1}$ of serum ACTH (with a sensitivity of $28.6 \%$; fig. 2 b). Conversely, serum cortisol and the PORT score can serve as indicators of the length of hospital stay (fig. 3a). The area under the ROC curve was 0.716 (95\% CI 0.540-0.892) for serum ACTH, 0.818 (95\% CI $0.626-1.010)$ for serum cortisol and 0.795 (95\% CI 0.559-1.030) for the PORT score. Specificity $>92.5 \%$ was achieved for $>29.7 \mu \mathrm{g} \cdot \mathrm{dL}^{-1}$ of serum cortisol (with a sensitivity of $57.1 \%$; fig. 3b). A significant relationship can be confirmed between each of serum ACTH, cortisol and the PORT score and the length of hospital stay in patients without administration of
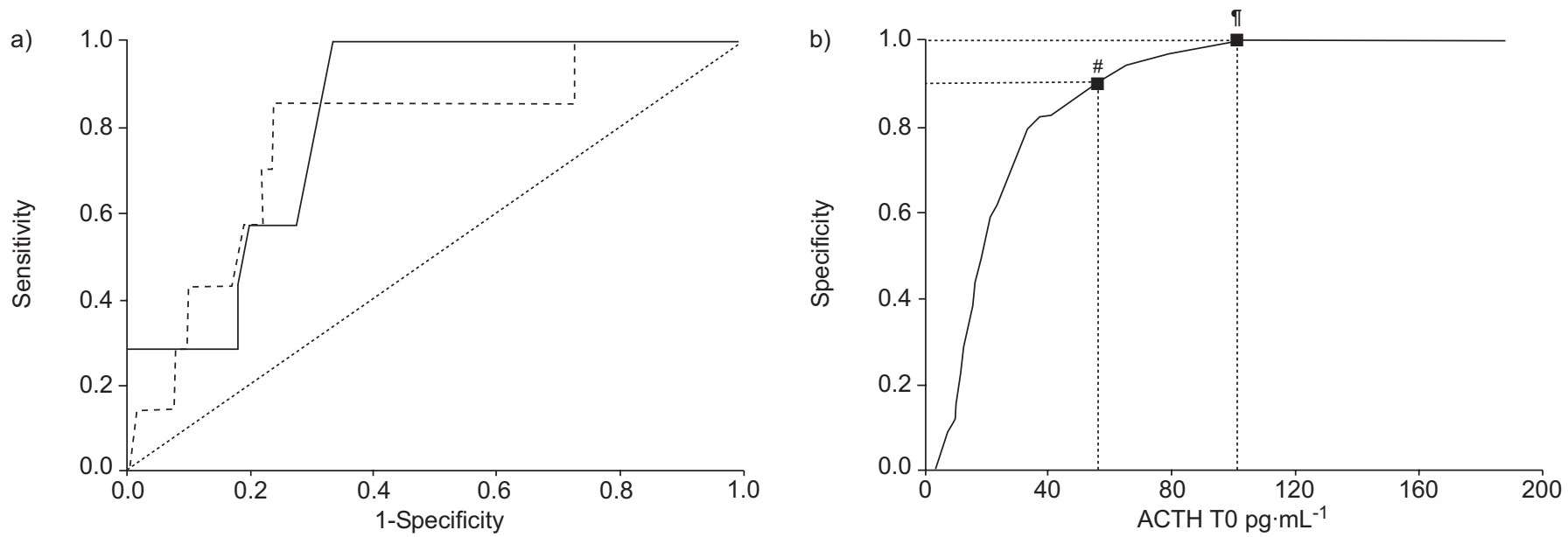

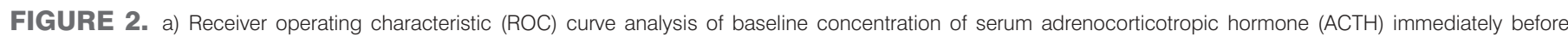

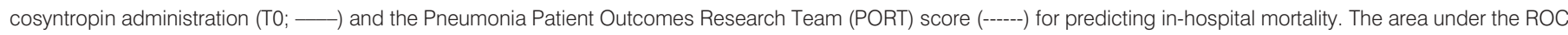

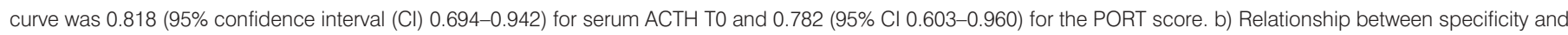
concentration of serum ACTH TO. ${ }^{*}$ : specificity=0.9; ${ }^{\bullet}$ : specificity=1.0 

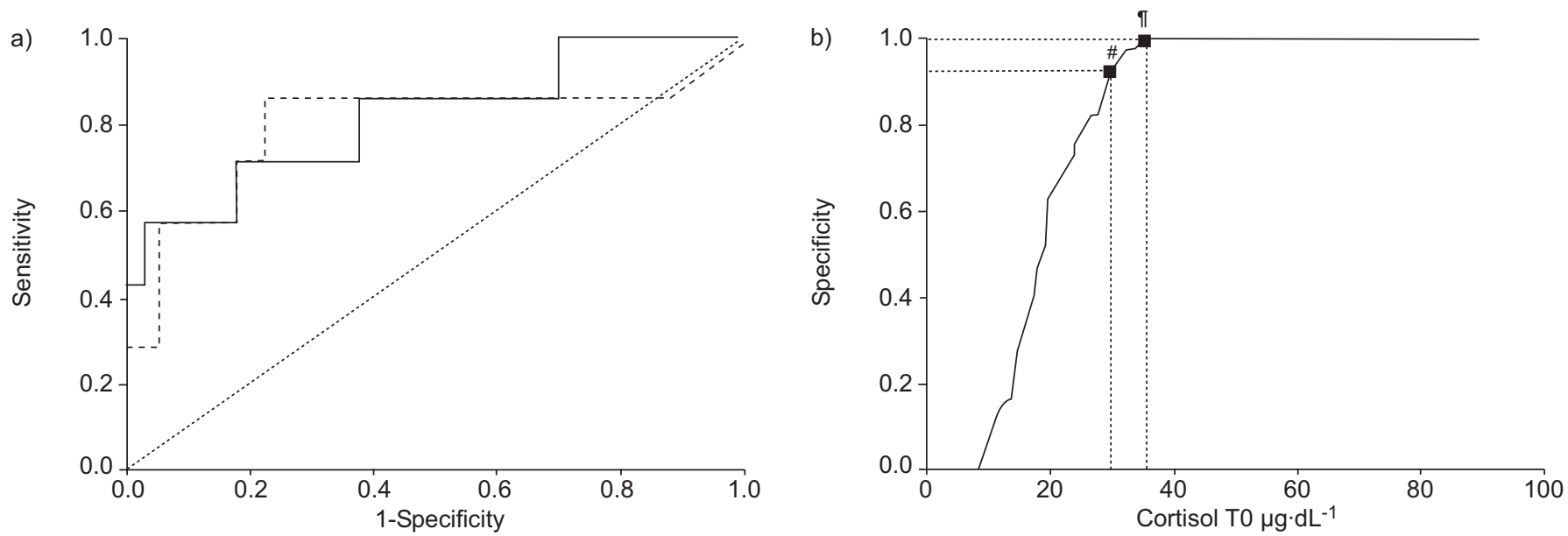

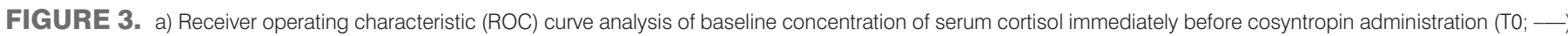

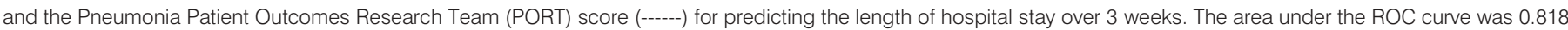

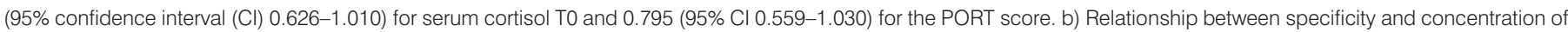
serum cortisol T0. ${ }^{\#}$ : specificity=0.9; ${ }^{\bullet}$ : specificity=1.0.

corticosteroids (data not shown). The effect of corticosteroids on the outcomes of in-hospital death and length of hospital stay was evaluated retrospectively, but no significant data were obtained (data not shown).

\section{DISCUSSION}

There have been only a few reports that investigated adrenal function in patients with CAP, including moderate-to-severe cases with septic shock, and there have been no previously published data about serum ACTH in patients with CAP. The present authors have shown that adrenal function was

\begin{tabular}{lc} 
TABLE 1 & $\begin{array}{c}\text { General characteristics of patients hospitalised } \\
\text { with community-acquired pneumonia }\end{array}$ \\
Subjects n & 64 \\
Sex male & $40(63)$ \\
Age yrs & $77(27-96)$ \\
PORT score & $110(0-207)$ \\
Pneumonia Severity Index & \\
I & $6(9)$ \\
II & $3(5)$ \\
III & $11(17)$ \\
IV & $23(36)$ \\
V & $21(33)$ \\
Comorbidity & \\
Heart failure & $11(17)$ \\
Liver disease & $8(13)$ \\
Septic shock & $7(11)$ \\
Malignancy & $7(11)$ \\
Cerebrovascular disease & $6(9)$ \\
Kidney disease & $6(9)$ \\
Steroid administration after stimulation test & $14(22)$ \\
Patients whose tests took place from 07:00-10:00 h & $54(84)$ \\
\hline
\end{tabular}

Data are presented as $\mathrm{n}(\%)$ or median (range), unless otherwise indicated. PORT: Pneumonia Patient Outcomes Research Team. correlated with the PORT score. In particular, the level of serum ACTH before stimulation by cosyntropin was significantly correlated with the number of in-hospital deaths, whereas the level of serum cortisol was significantly correlated with the length of hospital stay. The PORT score correlated not only with the number of in-hospital deaths, but also with the length of hospital stay. It was found that the level of serum ACTH or cortisol on admission or the morning after admission becomes positively correlated with the severity of CAP. This result in the present study of hospitalised patients with CAP is consistent with a previous study of 189 patients with septic shock [1] and with a recent report by CHRIST-CRAIN et al. [14]. However, the present study provides additional information about serum ACTH levels in patients with CAP.

In a previous report by ANNANE et al. [1], the survival curves for baseline serum cortisol and the increase of serum cortisol after cosyntropin stimulation were analysed. The group with baseline serum cortisol $\leqslant 34 \mu \mathrm{g} \cdot \mathrm{dL}^{-1}$ showed a significantly greater probability of survival than that with baseline serum cortisol $>34 \mu \mathrm{g} \cdot \mathrm{dL}^{-1}(\mathrm{p}<0.001)$. In addition, the group with the increase of serum cortisol $>9 \mu \mathrm{g} \cdot \mathrm{dL}^{-1}$ showed significantly larger probability of survival than the group with an increase of serum cortisol $\leqslant 9 \mu \mathrm{g} \cdot \mathrm{dL}^{-1}(\mathrm{p}<0.001)$.

CHRIST-CRAIN et al. [14] have investigated the predictive values of serum total cortisol, free cortisol, procalcitonin, C-reactive protein, leukocytes and Pneumonia Severity Index (PSI) for the outcome of death. Serum total cortisol and free cortisol were reported to increase as PSI increased, and both total and free cortisol levels in patients who died during follow-up were significantly higher than in survivors. In the present study, serum free cortisol could not be examined as it could not be measured in the present authors' hospital. Serum free cortisol is reported to be a more precise marker of adrenal function in patients with hypoproteinaemia, especially in patients whose serum albumin is $\leqslant 2.5 \mathrm{~g} \cdot \mathrm{dL}^{-1}$ [7]. Instead, serum ACTH was measured and cosyntropin stimulation tests were performed. This is a classically used method to check adrenal function, 


\begin{tabular}{|c|c|c|}
\hline TABLE 2 & \multicolumn{2}{|c|}{$\begin{array}{l}\text { Type of pathogen and antimicrobial agents used } \\
\text { in patients with community-acquired pneumonia }\end{array}$} \\
\hline \multirow{2}{*}{\multicolumn{3}{|c|}{$\begin{array}{l}\text { Subjects } n \\
\text { Pathogen }\end{array}$}} \\
\hline & & \\
\hline \multicolumn{2}{|c|}{ Streptococcus pneumoniae } & $9(14)$ \\
\hline \multicolumn{2}{|c|}{ Haemophilus influenzae } & $7(11)$ \\
\hline \multicolumn{2}{|c|}{ Klebsiella pneumoniae } & $6(9)$ \\
\hline \multicolumn{2}{|c|}{ Mycoplasma spp. } & $2(3)$ \\
\hline \multicolumn{2}{|c|}{ Chlamydia spp. } & $2(3)$ \\
\hline \multicolumn{2}{|c|}{ Legionella spp. } & $2(3)$ \\
\hline \multicolumn{2}{|c|}{ Brahamella catarrhalis } & $1(2)$ \\
\hline \multicolumn{2}{|c|}{ Escherichia coli } & $1(2)$ \\
\hline \multicolumn{2}{|l|}{ MRSA } & $1(2)$ \\
\hline \multicolumn{2}{|c|}{ Unknown pathogen } & $33(52)$ \\
\hline \multicolumn{3}{|c|}{ Antimicrobial agents } \\
\hline \multicolumn{2}{|c|}{ Cephalosporins (third generation) + non $\beta$-lactum agents ${ }^{\#}$} & $28(44)$ \\
\hline \multicolumn{2}{|c|}{ Cephalosporins (third generation) } & $8(13)$ \\
\hline \multicolumn{2}{|c|}{ Ampicillin/sulbactam } & $8(13)$ \\
\hline \multicolumn{2}{|c|}{ Cephalosporins (fourth generation) + non $\beta$-lactum agents ${ }^{\#}$} & $7(11)$ \\
\hline \multicolumn{2}{|c|}{ Ampicillin/sulbactam + non $\beta$-lactum agents ${ }^{\#}$} & $5(8)$ \\
\hline \multicolumn{2}{|c|}{ Piperacillin/tazobactam } & $2(3)$ \\
\hline \multicolumn{2}{|c|}{ Carbapenem + non $\beta$-lactum agents ${ }^{\#}$} & $2(3)$ \\
\hline \multicolumn{2}{|c|}{ Cephalosporins (fourth generation) } & $1(2)$ \\
\hline \multicolumn{2}{|c|}{ Piperacillin/tazobactam + non $\beta$-lactum agents ${ }^{\#}$} & $1(2)$ \\
\hline \multicolumn{2}{|c|}{ Carbapenem } & $0(0)$ \\
\hline \multicolumn{2}{|l|}{ Other } & $2(3)$ \\
\hline
\end{tabular}

Data are presented as $n(\%)$, unless otherwise stated. MRSA: methicillinresistant Staphylococcus aureus. \#: non $\beta$-lactum agents include minocycline, fluoroquinolone, macrolide and clindamycin.

especially in critically ill patients $[1,4,6]$. Interestingly, the present data suggest that serum ACTH could be the predictor of death in patients with CAP and that the cosyntropin stimulation test did not serve well to predict outcomes even when considering the result that the number of patients who had serum albumin $\leqslant 2.5 \mathrm{~g} \cdot \mathrm{dL}^{-1}$ was only two. HAMRAHIAN et al. [7] have shown that serum ACTH increases in critically ill patients, while no data are documented in a population of CAP. This is thought to be due to the activation of the HPA axis. In stressful conditions, cytokines such as interleukin (IL)-1, IL- 6 and tumour necrosis factor- $\alpha$ released from the site of injury or after exposure to endotoxin activate the HPA axis by stimulating the classical pathway of corticotropin-releasing hormone and ACTH secretion $[19,20]$.

The diagnosis of adrenal insufficiency in critically ill patients, also termed "critical illness-related corticosteroid insufficiency" (CIRCI) [21], has long been difficult to define, but some data have been reported by ANNANE et al. [6]. It has been suggested that CIRCI is likely when baseline serum cortisol level is $\leqslant 10 \mu \mathrm{g} \cdot \mathrm{dL}^{-1}$ or when the increase of serum cortisol after cosyntropin stimulation is $\leqslant 9 \mu \mathrm{g} \cdot \mathrm{dL}^{-1}$, whereas it is unlikely when the cortisol level after stimulation is $>44 \mu \mathrm{g} \cdot \mathrm{dL}^{-1}$ or when the cortisol increase is $>16.8 \mu \mathrm{g} \cdot \mathrm{dL}^{-1}$. ANNANE [6] suggested that the methyrapone test might be a useful tool for diagnosis of CIRCI. In the present study, nine patients met the criteria, three of which were in septic shock. For the other six patients, who were not in septic shock, it is unclear whether they suffered from

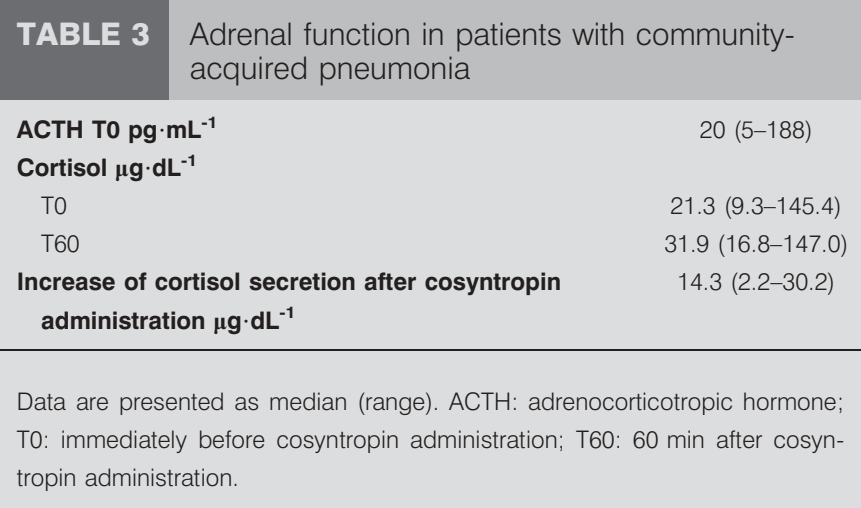

CIRCI as the methyrapone test was not performed, the diagnostic criteria were constructed from the study of patients with septic shock and patients lacked the specific features of adrenal insufficiency, such as hypoglycaemia, hypotension and increased eosinophil count.

Furthermore, the present results gave new insights into the concept of CIRCI. The fact that the severity of CAP increased as the levels of serum cortisol and ACTH increased has been disclosed, while no patients in the present study showed baseline cortisol levels of $\leqslant 10 \mu \mathrm{g} \cdot \mathrm{dL}^{-1}$. This may suggest that such CIRCI in CAP might be rare, although the dysfunction of HPA axis is correlated with the severity of CAP. This observation is contrary to the previous reports that evaluated patients with severe sepsis and septic shock [4-6] and with severe CAP [10], but was consistent with the report of CHRISTCRAIN et al. [14]. What caused this discrepancy? One interpretation is that the subject population of former studies was different from that reported by CHRIST-CRAIN et al. [14] and from the present study population in that more severely ill patients who needed intensive care were included in the former studies.

According to previous studies, most critically ill patients, including those who suffered from severe sepsis but were not in septic shock, were considered to have dysfunction of HPA axis, which is often called "adrenal endocrinological stress" [22]. The present authors suggest that there might be some critical turning point from dysfunction of HPA axis to CIRCI. It has also been suggested that CIRCI might be rare in patients with CAP and that the severity of CAP might be predicted significantly by the dysfunction of HPA axis rather than by CIRCI.

The limitation of the present study is that it was of a small sample size in a single institution; therefore, there was only a small number of in-hospital deaths. This might be the reason why a significant correlation could not be observed in survival with the level of baseline serum cortisol or the increase of serum cortisol after cosyntropin stimulation. The efficacy of steroid replacement therapy for CAP with severe sepsis or septic shock has been supported in previous studies $[12,17,18]$ and even recommended in the 2007 Infectious Diseases Society of America/American Thoracic Society consensus guidelines on the management of CAP in adults [23], but criteria for steroid replacement therapy is controversial and it is still unknown whether CIRCI is preceded by severe pneumonia or 
vice versa. It is believed that the adrenal function of patients with CAP should be investigated in a larger population, and criteria for steroid replacement therapy should be established from the viewpoint of the risk and benefit of steroid administration, as the CORTICUS study reported that hydrocortisone therapy increased the relative risk ratio of complications such as new sepsis and septic shock [9].

In conclusion, the present study showed that serum cortisol and serum adrenocorticotropic hormone were correlated with the Pneumonia Patient Outcomes Research Team score. In particular, both of these were correlated with clinical outcomes such as the number of in-hospital deaths and the length of hospital stay. Adrenal function may play a significant role in predicting the severity of community-acquired pneumonia.

\section{ACKNOWLEDGEMENTS}

The authors would like to thank M. Tsukamoto (St Luke's International Hospital, Tokyo, Japan) and H. Ueda (Yamaguchi Red Cross Hospital, Yamaguchi, Japan) for clinical support with enrolling patients, and J. Levi Joshua (St Luke's International Hospital) for preparing this manuscript.

\section{REFERENCES}

1 Annane D, Sébille V, Charpentier C, et al. Effect of treatment with low doses of hydrocortisone and fludrocortisone on mortality in patients with septic shock. JAMA 2002; 288: 862-871.

2 Annane D, Bellissant E, Bollaert PE, Briegel J, Keh D, Kupfer Y. Corticosteroids for treating severe sepsis and septic shock. Cochrane Database Syst Rev 2004; 1: CD002243.

3 Minneci PC, Deans KJ, Banks SM, Eichacker PQ, Natanson C. Meta-analysis: the effect of steroids on survival and shock during sepsis depends on the dose. Ann Intern Med 2004; 141: 47-56.

4 Annane D, Sébille V, Troché G, Raphaël JC, Gajdos P, Bellissant E. A 3-level prognostic classification in septic shock based on cortisol levels and cortisol response to corticotropin. JAMA 2000; 283: 1038-1045.

5 Sibbald WJ, Short A, Cohen MP, Wilson RF. Variations in adrenocortical responsiveness during severe bacterial infections. Unrecognized adrenocortical insufficiency in severe bacterial infections. Ann Surg 1977; 186: 29-33.

6 Annane D, Maxime V, Ibrahim F, Alvarez JC, Abe E, Boudou P. Diagnosis of adrenal insufficiency in severe sepsis and septic shock. Am J Respir Crit Care Med 2006; 174: 1319-1326.

7 Hamrahian AH, Oseni TS, Arafah BM. Measurements of serum free cortisol in critically ill patients. $N$ Engl J Med 2004; 350: 1629-1638.

8 Ligtenberg JJ, Zijlstra JG. The relative adrenal insufficiency syndrome revisited: which patients will benefit from lowdose steroids? Curr Opin Crit Care 2004; 10: 456-460.
9 Sprung CL, Annane D, Keh D, et al. Hydrocortisone therapy for patients with septic shock. N Engl J Med 2008; 358: 111-124.

10 Salluh JI, Verdeal JC, Mello GW, et al. Cortisol levels in patients with severe community-acquired pneumonia. Intensive Care Med 2006; 32: 595-598.

11 Feldman C, Joffe B, Panz VR, et al. Initial hormonal and metabolic profile in critically ill patients with communityacquired lobar pneumonia. S Afr Med J 1989; 76: 593-596.

12 Confalonieri M, Urbino R, Potena A, et al. Hydrocortisone infusion for severe community-acquired pneumonia: a preliminary randomized study. Am J Respir Crit Care Med 2005; 171: 242-248.

13 Oppert M, Schindler R, Husung C, et al. Low-dose hydrocortisone improves shock reversal and reduces cytokine levels in early hyperdynamic septic shock. Crit Care Med 2005; 33: 2457-2464.

14 Christ-Crain M, Stolz D, Jutla S, et al. Free and total cortisol levels as predictors of severity and outcome in communityacquired pneumonia. Am J Respir Crit Care Med 2007; 176: 913-920.

15 Fine MJ, Auble TE, Yealy DM, et al. A prediction rule to identify low-risk patients with community-acquired pneumonia. N Engl J Med 1997; 336: 243-250.

16 Miyashita N, Matsushima T, Oka M, Japanese Respiratory Society. The JRS guidelines for the management of community-acquired pneumonia in adults: an update and new recommendations. Intern Med 2006; 45: 419-428.

17 Montón C, Ewig S, Torres A, et al. Role of glucocorticoids on inflammatory response in nonimmunosuppressed patients with pneumonia: a pilot study. Eur Respir J 1999; 14: 218-220.

18 Marik P, Kraus P, Sribante J, Havlik I, Lipman J, Johnson DW. Hydrocortisone and tumor necrosis factor in severe community-acquired pneumonia. A randomized controlled study. Chest 1993; 104: 389-392.

19 Chrousos GP. The hypothalamic-pituitary-adrenal axis and the immune-mediated inflammation. N Engl J Med 1995; 332: 1351-1362.

20 Bornstein SR, Chrousos GP. Clinical review 104: Adrenocorticotropin (ACTH)- and non-ACTH-mediated regulation of the adrenal cortex: neural and immune input. J Clin Endocrinol Metab 1999; 84: 1729-1736.

21 Marik PE. Mechanisms and clinical consequences of critical illness associated adrenal insufficiency. Curr Opin Crit Care 2007; 13: 363-369.

22 Cooper MS, Stewart PM. Corticosteroid insufficiency in acutely ill patients. $N$ Engl J Med 2003; 348: 727-734.

23 Mandell LA, Wunderink RG, Anzueto A, et al. Infectious Diseases Society of America/American Thoracic Society consensus guidelines on the management of communityacquired pneumonia in adults. Clin Infect Dis 2007; 44: Suppl. 2, S27-S72. 